\title{
3D MODELING AND DYNAMIC CHARECTARIZATION OF STEAM TURBINE PACKET BLADE AND CYCLIC SYMMETRIC ANALYSIS
}

\author{
T.S.S.R.Krishna ${ }^{1}$, Lakshmana Kishore. $\mathbf{T}^{2}$ \\ ${ }^{1}$ M.Tech (student), Mechanical Engineering Department, GIET, Andhra Pradesh, India, tssrkrishna.316@gmail.com \\ ${ }^{2}$ Associate Professor, Mechanical Engineering Department, GIET, Andhra Pradesh, India, tlkishore.giet@gmail.com
}

\begin{abstract}
A rotating turbine blade (bucket) is the component, which converts the kinetic energy of the flowing fluid into mechanical energy. Thus the reliability of these blades is very important for the successful operation of a turbine. Turbine blades experience fluctuating forces when they pass through non-uniform fluid flow from the stationary vanes. The basic design consideration is to avoid or to minimize the dynamic stresses produced by the fluctuating forces. The aim of the project is to estimate the stresses occurring in the packet blade of steam turbine in last stage due to centrifugal forces and steam bending forces. The blade, root and disc are modeled separately in ANSYS package and they are assembled using constrained equations. After giving the constrain equations cyclic symmetric analysis conditions are applied and then static and model analysis are carried out. After that Campbell and safe diagrams are plotted.
\end{abstract}

Index Terms: ANSYS, Campbell diagrams, Cyclic symmetric analysis and Safe diagrams.

\section{INTRODUCTION}

The break down and failures of turbine machineries have been influencing such as coseq1uential damages, hazards to public life and most importantly the cost repairs. To avoid these, it is obvious that the blade of turbo machinery must be made structurally stronger, that means not in dimensions and/or use of materials of construction, but keeping the operating stresses well within the limits.

Turbo machinery blades are classified into two categories depending on their manner of operation as either impulse or reaction blades.

Reasons for failure of bladed disc:

- Excessive stresses

- Resonance due to vibration

- Operating environmental effects.

Ever increasing demands of high performance together with reliability of operation, long life and light weight necessitate consistent development of almost every part of steam turbine blades from a vital part of a turbo machine. Apart from their shape and geometry, on which the performance characteristics of the machine largely depend, their dynamic strength is of considerable importance as far as the reliability operation and life of the engine are concerned.

High cycle fatigue plays a significant role in many turbine blade failures. During operation, periodic fluctuations in the steam force occur at frequencies corresponding to the operating speed and harmonics and cause the bladed disk to vibrate.

The basic design consideration is to avoid or to minimize the dynamic stresses produced by the fluctuating forces.

\subsection{Failures in steam turbine blades}

$>$ Excessive stress

The total stress at any location of the blade is sum of the centrifugal tension, centrifugal bending, steady steam bending and the alternating bending. The amplitude of the alternating bending depends on the dynamic bending force, damping factor and the resonant frequency. Each of these is briefly discussed below to highlight their importance.

\section{$>$ Centrifugal stress}

In steam turbine, centrifugal stress is never the main cause of a blade failure, except in the rare cases of turbine runaway or due to low cycle fatigue caused by frequent start ups/shut downs. However, centrifugal stress is an important contributing factor with fatigue failure, corrosion fatigue failure and stress corrosion failures. The level of centrifugal stress is kept at such a level so as to have enough margins for alternating stress. The blade configuration is designed so as to keep the center of gravity of shroud, airfoil and root attachments, on a common radial axis. This prevents centrifugal induced torsion stresses. Using any of the standard FEM packages can best carry out analysis of centrifugal stress. 
$>$ Steam induced stress - steady state

Steam being the driving force, exerts loads on the blade due to steam pressure from the pressure side of the blade profile thereby inducing bending stresses. This bending stress is superimposed on the centrifugal tension. Although the net value of steady bending load and centrifugal stress alone is not normally a cause of blade failure, this is the basis for the majority of failures arising due to vibratory stresses superimposed upon them.

$>$ Steam induced stress - Alternating

Steam induced alternating stress can be induced by interrupted arc of admission, nozzle wake at nozzle passing frequency, mismatch of diaphragm nozzle at horizontal split and/or missing diaphragm blades, nozzle pitch variation, poor nozzle profile etc. Estimation of the vibratory stress caused by these requires the analysis of the response of the blade to the excitation forces caused by these. Obviously the inputs to such an analysis are the excitation levels, the damping, natural frequency and the mode shape of the blade vibration.

$>$ Resonant vibration

Vibration is important in designing turbine blades/disk since resonant vibratory stresses, sustained over a period of time, can cause fatigue failures. The period of time need not be large since a $500 \mathrm{~Hz}$ vibration accumulates 2 million cycles in an hour and 108 cycles in 2 days. Bladed disk poses a worst vibratory fatigue problem since it is directly exposed to a wide range of aerodynamic excitation and failure can result when any of the following matching takes place.

\subsection{Shrouds and packets:}

Shrouds are used to connect adjacent blades and the normal practice is to use discrete shroud segments to join a group of blades into packets as shown in fig 1.1 and 1.2 one advantage of packet is that it constrains the tip vibratory displacement and hence it lowers the vibratory stresses. The major advantage of a packet comes from the fact that there being a phase lag in the excitation acting on adjacent blades in packet, energy input over the entire packet can be low. Thus careful packet design can control the net energy input and hence can make resonance in the operating range tolerable.

For this blade packet of blades are modeled by joining 8 blades. Total number of blades is 280 with 34 packets

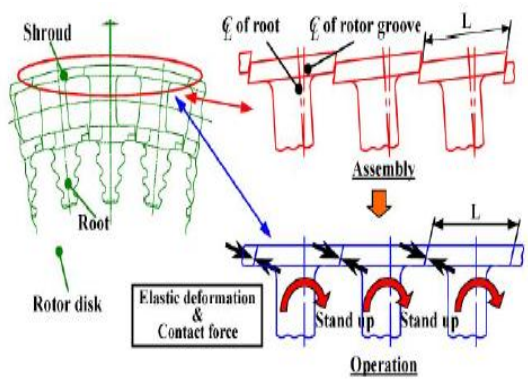

Fig 1: Advanced shroud blade

\section{THEORY OF STEAM TURBINE BLADES}

A rotating turbine blade is the component, which converts the energy of the flowing fluid into mechanical energy. Thus the reliability of these blades is very important for the successful operation of a turbine. Metallurgical examinations of failed blades show that almost all the failures can be attributed to the fatigue of metal. Blade failures due to fatigue are predominately vibration related.

Turbine vibration is known to be caused by several mechanisms, but sometimes failures of blade occur which cannot be explained by these mechanisms. These blades are called "rogue blades". However, studies have shown that these failures were caused by abnormally high stresses. The consequences of these discoveries have lead to extensive studies of bladed disc vibration characteristics.

It has been suggested that these high stresses in a particular blade might be influenced by these factors. One factor is the non-uniform circumferential fluid flow and gear meshing. These may excite a particular blade or packet of blades to vibrate at high stress levels leading to fatigue failure. The second factor is the flexibility of the disc. Due to this flexibility, forces may be transmitted between any blade and its neighbors'. This inter-blade coupling may thus excite a particular blade or a group of blades thus causing failures due to extensive fatigue. The last factor is that blades are, in practice, slightly different, with the consequence that their natural frequencies differ with a particular rotor speed; some of these blades may be excited, with consequence that they fail due to fatigue.

\subsection{Design procedure:}

A turbo machine blade is usually a cantilever beam or plate is tapered and twisted with an airfoil cross-section. Typically a turbo machine has several stages, each stage with a stator and rotor. In the stator, they are all inserted as diaphragms or nozzles in a ring to guide the flow medium at an appropriate entry angle into rotor blades. The rotor blades are mounted on a disc at a stagger angle to the machine axis and they convert the thermal energy into mechanical energy in turbine. In turbine steam enters at high pressure and temperature in the 
first stage and expands while passing through the several stages before it is let out from the last stage with low temperature and pressure after extracting as much as thermal energy as possible. Hence, the short blades in high pressure have high frequency of the order of $1000 \mathrm{~Hz}$ which becomes progressively lower about $100 \mathrm{~Hz}$ in the last stage long blades. In the compressor stage, the operation principle is reversed to compress the gases utilizing the supplied mechanical power.

A good design of turbine blade thus consists of the following steps:

- Determination of natural frequencies and mode shapes.

- Determination of non-steady forces due to stage flow interaction.

- Evaluation of damping and generating appropriate models.

- Modal analysis and determination of dynamic stresses.

- Life estimation based on cumulative damage fatigue theories.

\subsection{Campbell Diagram:}

The Campbell diagram is a pictorial representation of the variation of blade fundamental and harmonic frequencies as a function of the speed of rotation. This diagram is used to determine the adequacy of a blade in avoiding operation at various conditions, where it could be operating at risk. The Campbell diagram or the interference diagram indicates the vibratory stress levels in a given stage. Since almost all the blade failures are caused by vibratory stress, much reliability conscious purchasers are requesting Campbell diagrams with turbine quotes or orders. A Campbell diagram is a graph with turbine speed (rpm) plotted on the horizontal axis and frequency in cycles per second plot on the vertical axis. The blade frequencies and the frequencies of exciting forces are plotted on the Campbell diagram. This diagram predicts where the blades natural frequencies coincide with the exciting frequencies. When a blade frequency and an exciting frequency are equal, or intersect, it is called a resonance.

\subsection{Safe Diagram:}

Evaluation of turbine blade disc region is done by using Campbell diagram. An alternative method of evaluation is Safe Diagram.

Campbell diagram is a two dimensional projection of a three dimensional surface, hence it does not retain all the information for evaluation. Another two dimensional projection of the same surface contains more information for easier evaluation. This diagram, refined for packeted bladed disc assembly by Dresser-Rand, is called safe diagram. the use of the safe diagram for a bladed disc assembly facilitates the design evaluation process. Usually Safe Diagram was drawn between nodal diameter and frequency and then it was taken into Campbell plane. It was a 3D-Diagram

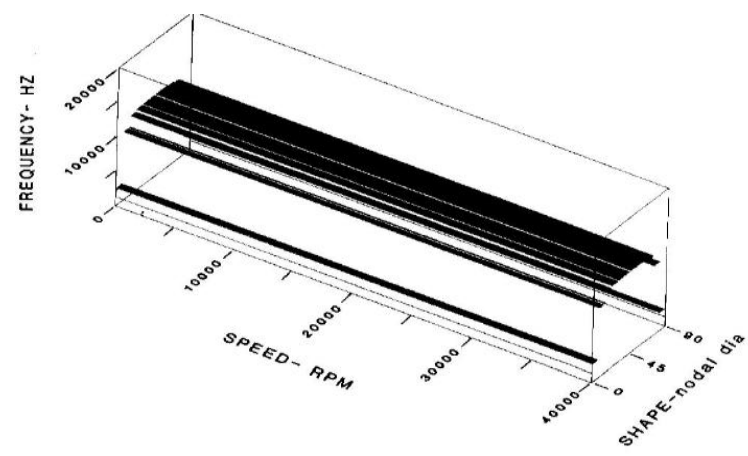

Fig 2: Safe diagram

\section{CYCLIC SYMMETRIC ANALYSIS USING}

\section{ANSYS:}

A CYCLIC symmetry analysis requires that you model a single sector, called the basic sector. A proper basic sector represents one part of a pattern that, if repeated $\mathrm{N}$ times in cylindrical coordinate space, yields the complete model, To carry cyclic symmetric analysis we have to give cyclic symmetric conditions to the existed sector. In cyclic condition if one node moves in a direction then all nodes connected to the node also move in the same direction so in cyclic symmetry we have to give coupling equations to the areas and volumes in the sector which have to be cyclic. For this model coupling equations are given to the side areas of shroud and disc sectors.

\subsection{FEM modal details:}

Assumptions

Material is isotropic.

Modulus of elasticity is $\quad 2.0 \mathrm{e} 5 \mathrm{~N} / \mathrm{mm} 2$.

Poisson's ratio is 0.3 .

Density of the material is $\quad 7.85 \mathrm{e}-9 \mathrm{Kg} / \mathrm{mm} 3$.

\subsection{Element type:}

The element used in discretization of the solid model is 8 nodded brick element, which has three translational degrees of freedom in $\mathrm{x}, \mathrm{y}$ and $\mathrm{z}$ direction.

\subsection{Modal analysis of packeted blade:}

The concept of packeted blade analysis is to simulate and analyze the single blade sector of particular as this form the basic behavior of the modal. Modal analysis is conducted for the single blade by running at different rpm. The blade is constrained for all degrees of freedom at the base of the blade where it is attached to the root and shroud. In this analysis the pre-stressed effect of the blade is also considered. Due to the 
self-weight when the blade is run at different speeds its natural frequencies varies.

\section{RESULTS \& DISCUSSIONS:}

After completing modal analysis at different operating speeds we can get frequencies for 3 modes of extraction. Due to these values we can draw Campbell diagrams.

Deformed and undeformed, Nodal solutions and Campbell diagrams for different operating speed and natural frequencies are shown in the below figs.

In the present work static and modal analysis are carried out at running speed 3000rpm.First static analysis was done at different frequency values. Figs. 3,4 Shows deformed and undeformed shapes of the blade sector model. In figs. 5,6,7,8,9 Shows von mios stress at different frequencies. By this we know maximum deformation and where the maximum stresses occur. For this model maximum stress occurs where root and disc joins by the pin. After completing static analysis at every frequency value we go for modal analysis at running speed 3000 rpm with 3 modes of extractions. The natural frequency values occur at different speeds were shown in the table.

By these values we draw Campbell diagram as shown in fig. 10. By this Campbell diagram we observe that mode shape lines are not crossing with engine order lines at running speed. Hence this model is safe at that running speed with cyclic symmetric conditions.

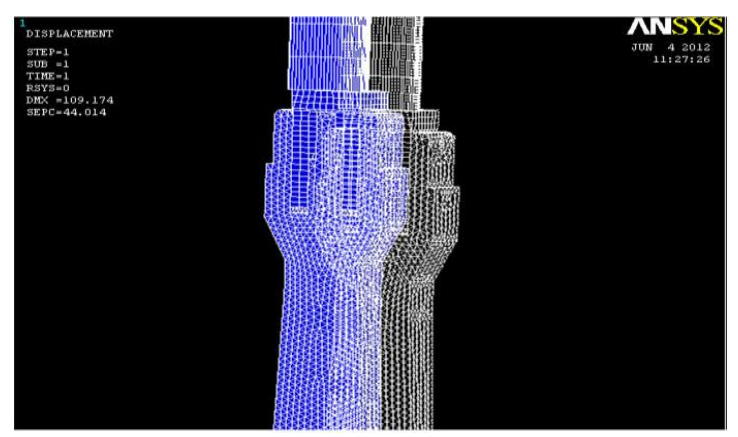

Fig.3 Deform and Undeform shape at 2000rpm

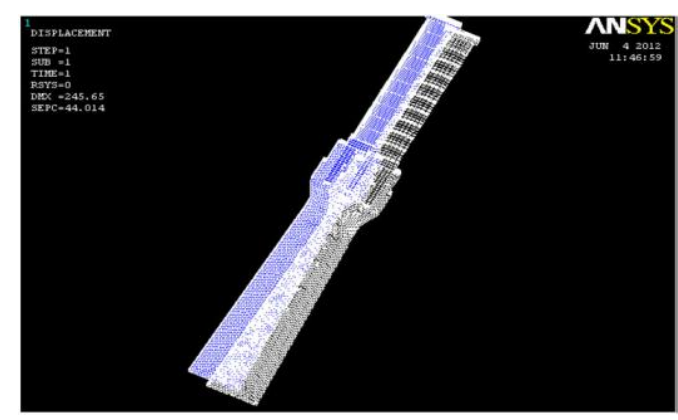

Fig.4 Deform and Undeform shape at 3000rpm

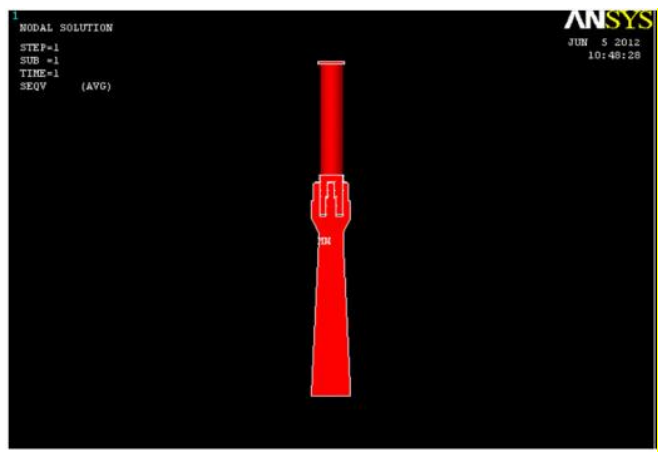

Fig.5 Von mios stress for $0 \mathrm{rpm}$

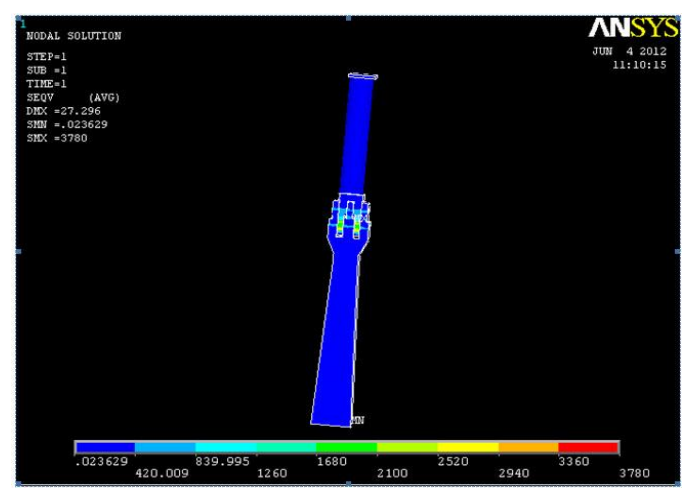

Fig.6 Von mios stress for 1000rpm

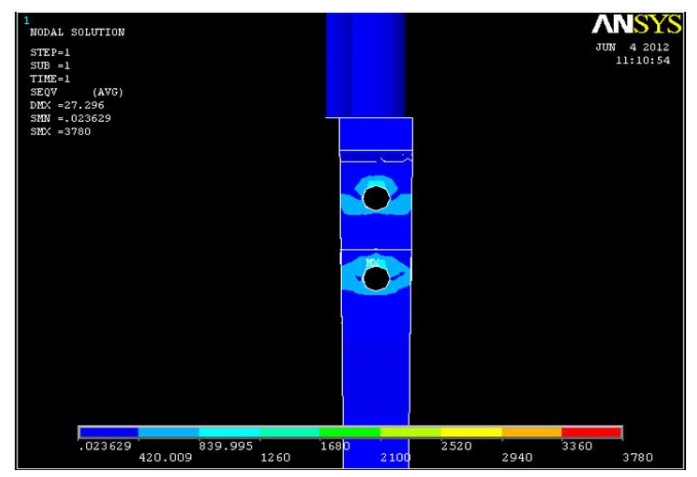

Fig.7 Von mios stress for 2000rpm

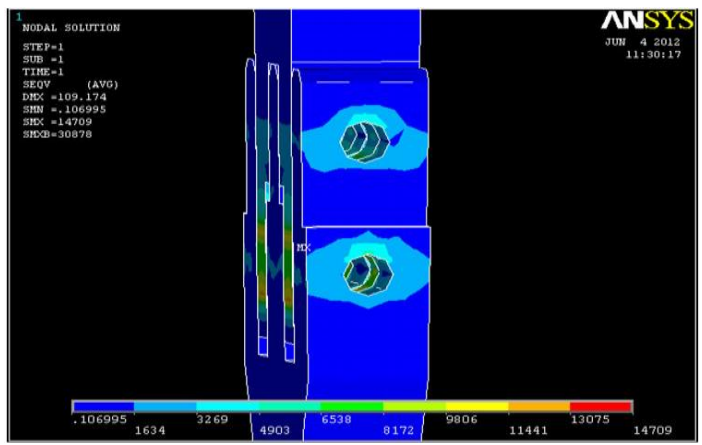

Fig.8 Von mios stress for 3000rpm 


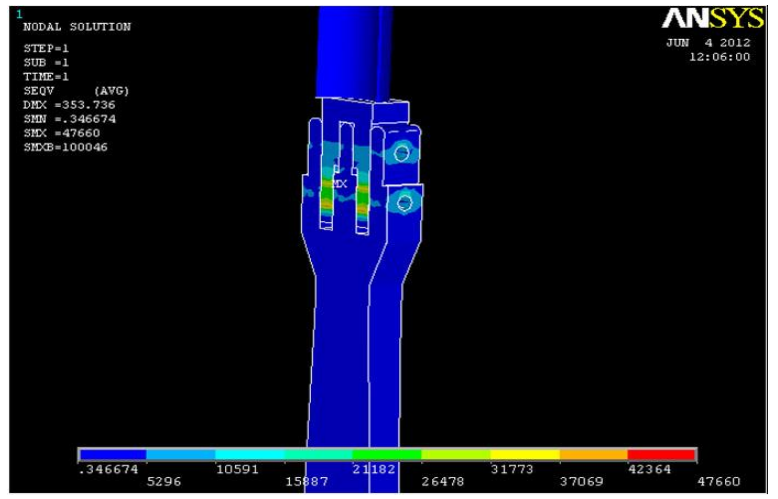

Fig.9 Von mois stress for 3600rpm

Table -1: Natural frequencies at different speeds

\begin{tabular}{|l|l|l|l|l|l|}
\hline $\begin{array}{l}\text { Freque } \\
\text { ncy }\end{array}$ & 0 & 1000 & 2000 & 3000 & 3600 \\
\hline Mode1 & 60.51 & 62.58 & 68.35 & 76.82 & 82.62 \\
& 9 & 4 & 7 & 1 & 9 \\
\hline Mode2 & 149.3 & 150.1 & 152.4 & 156.2 & 159.1 \\
& 7 & 2 & 8 & 5 & 1 \\
\hline Mode3 & 216.7 & 214.2 & 206.2 & 191.2 & 178 \\
& 3 & 5 & 7 & 9 & \\
\hline
\end{tabular}

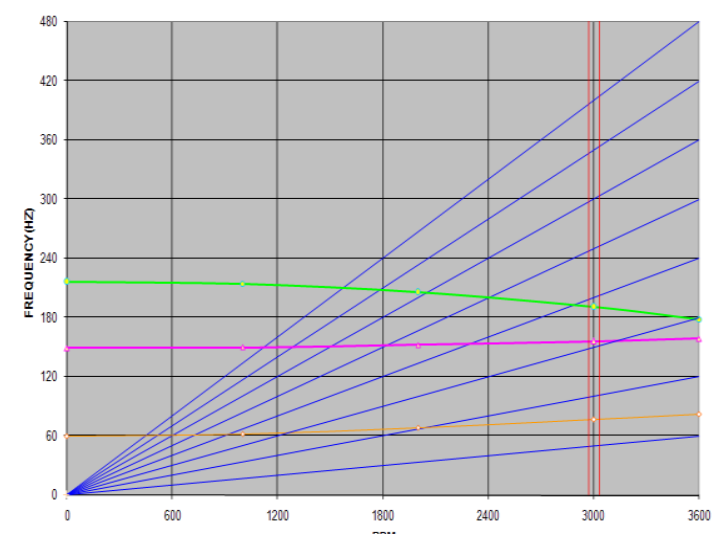

Fig.10 : Campbell diagram of blade at different speeds

\section{CONCLUSIONS}

The main objective of this project is to reduce the failures of turbine blade to avoid dynamic stresses vibration caused failures and fatigue failures.

Modeling of packeted blade was completed. A single blade sector from the packet was taken and modal analysis at different operating speeds with cyclic symmetric conditions was carried out. With natural frequencies occurred at different speeds Campbell diagrams are plotted.

\section{SCOPE FOR FUTURE WORK:}

In the present work static and model analysis was done at cyclic conditions. In the future we can also draw safe diagram at this conditions by obtaining nodal diameters and nodal circles in cyclic symmetric analysis. These nodal diameters and nodal circles are obtained by giving cyclic expansions and cyclic options to the present sector in ANSYS.

\section{REFERENCES:}

[1] Yasutomo Kaneko, Kazushi Mori. Analysis of vibratory stress of integral shroud blade for mechanical drive steam turbine.

[2] N.S.Vyas, Sidharth, J.S.Rao, Dynamic stress analysis and a fracture mechanics approach to life prediction of turbine.

[3] J.S.Rao, K.CH.Periah, Estimation of Dynamic Stresses in Last Stage Steam Turbine Blades under Reverse Flow Conditions.

[4] J. Szwedowicz, R. Visser Paper on Nonlinear Forced Vibration of shrouded turbine blades.

[5] Frank Kushner Rotating component modal analysis and resonance avoidance recommendations

[6] Rzadkowski Drewczynski, Coupling of Vibration of several Bladed discs on the shaft. 